DIANA VINASCO MARTÍNEZ*

Universidad Icesi (Cali, Colombia)

\title{
Transporte público en Cali: aspectos generales de su configuración en el siglo $\mathrm{XX}^{* *}$
}

\author{
Public transport in Cali: general feautures \\ of its configuration in the twentieth century \\ Transporte público em Cali: aspectos gerais \\ de sua configuração no século $X X$
}

\footnotetext{
* Licenciada en Historia de la Universidad del Valle. Estudiante becaria de la Maestría en Estudios sociales y políticos, Universidad Icesi, Cali, Colombia; y asistente de investigación del Centro de Estudios Afrodiaspóricos (CEAF) de la misma Universidad. Correo electrónico: dyvinasco@icesi.edu.co | ORCID: oooo-0oo2-0580-116o

** El presente artículo forma parte del trabajo de investigación sobre Transporte público, expansión urbana y planeación: Cali 1910-1993, realizado en la Universidad del Valle entre el 2015 y 2016. Artículo de investigación recibido el 21-10-2016 y aceptado $17-02-2017$.
} 


\section{Cómo citar}

VINASCO MARTÍNEZ, D. (2017). Transporte público en Cali: aspectos generales de su configuración en el siglo XX. Revista CS, 21, pp. 41-67. Cali, Colombia: Facultad de Derecho y Ciencias Sociales, Universidad Icesi.

DOI: http://dx.doi.org/10.18046/recs.i21.2295 
Resumen

Abstract

Resumo

El siguiente artículo describe el desarrollo del transporte público urbano en Cali durante el siglo XX a partir de la revisión de diversas fuentes documentales. Primero, se describe el surgimiento del transporte público urbano en la ciudad y su crecimiento en las primeras décadas del siglo XX. Luego se analiza el papel que el sector del transporte público adquirió en la configuración de la ciudad y de sus zonas periféricas, así como los intentos estatales por controlarlo o modificarlo. Finalmente, se ofrecen unas conclusiones sobre la persistencia de un único modelo de transporte público durante todo el siglo XX en Cali y sobre la importancia de los transportadores en la configuración de la ciudad.

PALABRAS CLAVE:

Transporte público | Cali | crecimiento urbano | siglo XX

The following article describes the development of urban public transportation in Cali during the twentieth century from the review of various documentary sources. First, the emergence of urban transport is described in the city and its growth in the first decades of the twentieth century. Then, the role the public transport sector acquired in shaping the city and its surrounding areas, as well as state attempts to control or modify analyzes. Finally, some conclusions about the persistence of a single model of public transportation are offered throughout the twentieth century in Cali and the importance of transporters in shaping the city.

\section{KEYWORDS:}

Public transport | Cali | urban growth | XX century 
O seguinte artigo descreve o desenvolvimento do transporte público urbano em Cali durante o século XX a partir da revisão de diversas fontes documentais. Primeiro, descreve-se o surgimento do transporte público urbano na cidade e seu crescimento nas primeiras décadas do século XX. Em seguida, analisa-se o papel que o setor de transporte público adquiriu na configuração da cidade e de suas zonas periféricas, bem como as tentativas estatais de controlá-lo ou modificá-lo. Finalmente, são oferecidas conclusões sobre a persistência de um único modelo de transporte público durante todo o século XX em Cali e sobre a importância dos transportadores na configuração da cidade.

PALAVRAS CHAVES:

Transporte público | Cali | crescimento urbano | século XX 


\section{Introducción}

Las problemáticas urbanas que actualmente enfrenta la ciudad de Cali, asociadas, entre otros fenómenos, a sus problemas de movilidad, plantean la necesidad de una reflexión sobre los cambios y continuidades que en materia de transporte público se han dado en la ciudad. El nuevo modelo de transporte público, basado en un sistema BRT, ha significado la emergencia de nuevas realidades en la movilidad de la ciudadanía caleña, que han terminado por revelar las problemáticas que se venían acrecentando desde el siglo pasado, con el aumento incontrolado de buses y empresas de transporte. Problemáticas que no están relacionadas únicamente con las deficiencias en el transporte, sino que están relacionadas con la imposibilidad de construir un proyecto de ciudad en el cual planeación no signifique corregir lo ya ejecutado.

Las transformaciones que la ciudad necesitaba no podían sustentarse únicamente en el sistema BRT implantado. En consecuencia, aunque el nuevo sistema no haya cumplido con las expectativas y a partir de su entrada en funcionamiento hayan surgido o aumentado algunas problemáticas; estas no pueden ser atribuidas exclusivamente a su llegada. Pues, a pesar de sus fallas, era necesario desde hace ya bastante tiempo. Siendo así, las reflexiones sobre el problema del transporte público en la actualidad, deberían indagar por las verdaderas causas de las dificultades de movilidad en la ciudad. Reflexionar sobre el desarrollo del transporte en Cali a través de su historia, quizás contribuya a entender las causas de las dificultades actuales.

Por ello, el presente artículo gira en torno a la pregunta por la importancia del transporte público en la ciudad de Cali en el siglo XX y de qué manera la configuración urbana de la ciudad incidió en su modelo de transporte. Para dar respuesta a estas inquietudes, se intenta comprender cómo surgió el modelo de transporte público de la ciudad, cuáles fueron sus características principales y qué propuestas institucionales fueron planteadas para transformarlo.

Para dicho propósito se recurrirá a una perspectiva de análisis histórico, más exactamente un enfoque de historia social, política e institucional, para el cual serán de gran importancia los conceptos de tiempo, espacio y sociedad sobre los cuales Édgar Vásquez establece una relación indisociable. Afirma el autor que la configuración física de un espacio urbano está determinada por las relaciones establecidas entre los grupos que lo habitan; sin embargo, el espacio no cumple un papel pasivo. Por el contrario, es el escenario que reproduce, condiciona y refleja las relaciones sociales. Por ello, cuando se generan cambios en las dinámicas sociales que hacen insuficiente el espacio urbano y su composición física, este comienza a ser transformado y adecuado por los actores sociales a sus nuevas necesidades (Vásquez, 1980:11).

En el mismo sentido, señala Jacques Aprile (1992), el concepto de formación espacial asocia tres categorías inseparables: tiempo, espacio y sociedad: «Por lo tanto, nacida de 
la formación social, la formación espacial es cambiante y se va modificando con ella y al ritmo impuesto por ella». En resumen, se pretende construir un modelo de análisis que logre evidenciar la relación indisociable que existe entre la expansión urbana, las políticas de planeación y el transporte público en la ciudad de Cali. Para lograr este objetivo, se realizará una breve descripción de los inicios del transporte público en la ciudad y las causas de su surgimiento; luego se intentará relacionar el transporte público con las dinámicas de explosión urbana ocurridas en la ciudad a partir de la década del cuarenta, y con las políticas de planeación que se implementaron en la ciudad y en el país. Finalmente, se analizará la evolución y crecimiento de las empresas de transporte y cómo sus dinámicas se relacionaron con la continua expansión de la ciudad y en tensión constante con las políticas de planeación que se pretendían aplicar en la ciudad.

\section{Inicios del transporte público}

El transporte público, exclusivamente urbano, había surgido a partir de la conformación del Barrio San Fernando en 1928, pues en ese momento la ciudad empezó a adquirir distancias que no era posible recorrer a pie. Con ello se dio el surgimiento de la primera empresa de buses urbanos, operada por la Compañía Comercial San Fernando, que era la encargada de ofrecer a los habitantes del nuevo barrio todas las comodidades para residir sin inconvenientes lejos del perímetro urbano, ya que se encontraban ubicados a unas 20 cuadras del área urbana de la ciudad y a 2,5 kilómetros del centro (Vásquez, 1995).

A finales de la década del veinte y la década del treinta, la mayoría de rutas tenían como principales puntos de origen-destino al barrio San Fernando y a los barrios El Peñón y Granada, sectores de clase media y alta, lo que evidencia que el transporte público en sus inicios tuvo como principal impulso a los barrios periféricos para familias con altos ingresos. La búsqueda realizada en el Diario Relator de los años 1928 a 1930, da cuenta de las siguientes rutas de buses: buses azules con ruta Estación-El Peñón; bus Carro Azul con ruta Plaza de Caicedo-San Fernando; buses amarillos con ruta Estación-Crucero-San Fernando; buses grises con ruta San Fernando-Plaza de Caicedo-Estación-Crucero; buses rojos, con dos rutas: El Peñón-Estación y El Peñón-Granada; todos los buses realizaban recorridos cíclicos (ver mapa 1). Algunos buses que también aparecen mencionados son: Embajador, Sucre, Gaviota y Santamaría, de los cuales no se mencionan rutas ni color.

La información sobre los buses y sus recorridos fue extraída de noticias referentes a accidentes de tránsito, protestas, solicitudes de vecinos y resoluciones de tránsito publicadas en Relator, pues la única empresa de transporte urbano que anunciaba sus servicios en los diarios era la Compañía Constructora, cuyos buses no eran promocionados con ningún color sino con el signo de la $\mathrm{Z}$ cruzada, lo que provocó que en 1938 
MAPA 1 Rutas de buses en Cali en la década de 1930

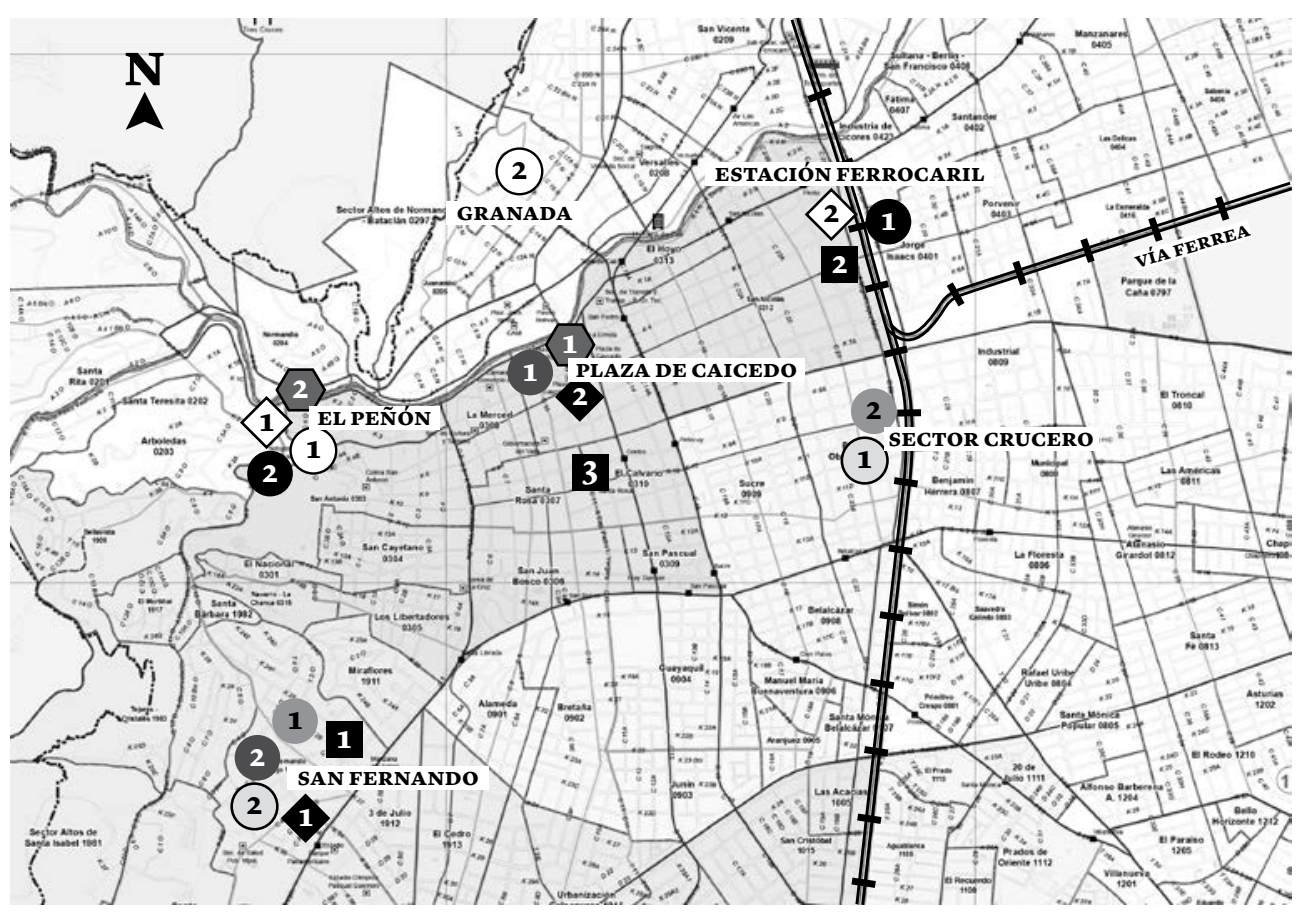

1 Punto de salida

2 Punto de retorno

3 Punto medio de la ruta

\begin{tabular}{lll}
\hline Ubicación & Línea o empresa & Ruta \\
\hline & Buses Azules & Estación del ferrocarril / Barrio El Peñon \\
\hline & Carro Azul & Plaza de Caicedo / Barrio San Fernando \\
\hline Buses Amarillos & Sector Crucero / Barrio San Fernando \\
\hline Buses Grises & Barrio San Fernando / Sector Crucero \\
\hline Buses Rojos & Barrio El Peñón / Estación del Ferrocarril \\
\hline Buses Rojos & Barrio El Peñón / Barrio Granada \\
\hline Comp. Construc. Colomb. & Barrio San Fernando / Plaza de Caicedo \\
\hline & Comp. Construc. Colomb. & Barrio San Fernando / Estación del Ferrocarril - pasando por la galería \\
\hline
\end{tabular}

Fuente: Elaboración propia a partir de la información recopilada en el diario Relator de los años 1928 a 1938. Plano tomado de la Subdirección de POT y Servicios Públicos, Departamento Administrativo de Planeación, Alcaldía de Cali, 2016. 
un movimiento antifascista caleño solicitara al Concejo multar a esta empresa, por utilizar una insignia del fascismo (Diario Relator, 1938). A pesar de que la información recopilada es escasa y fragmentada, arroja algunos indicios sobre la configuración de los recorridos, los principales puntos de origen-destino y el aumento vertiginoso de rutas a la par con el crecimiento de la ciudad.

El crecimiento del sector del transporte público provocó el surgimiento de diversas problemáticas relacionadas con la prestación del servicio a los usuarios y con las condiciones laborales de los conductores. Prueba de ello son las huelgas y paros llevados a cabo por el gremio de los trasportadores en las décadas del 30 y 40, entre las que se destacan por su magnitud, duración e importancia para los sectores políticos y económicos de la ciudad, el paro de transportadores de 1938. Se sumaban las continuas quejas sobre la mala prestación del servicio, la falta de «educación» de los conductores y la falta de control por parte de las autoridades municipales.

Luego de dos décadas en las que el transporte público urbano había venido configurándose en Cali, el Concejo de la ciudad decidió iniciar un proyecto para su municipalización en 1946, a través del Acuerdo 107 sobre la creación de una Junta Pro-municipalización del transporte que se encargaría de establecer un servicio municipal de buses en cooperación con las empresas de transporte existentes. El informe de comisión para el estudio del proyecto de Acuerdo, señaló:

Durante muchos años, casi desde la fecha de la motorización de los transportes, la ciudadanía ha reclamado insistentemente por el establecimiento de un sistema de transporte eficaz, propio de una ciudad moderna, libre de demoras y embarazos. Siendo, como es, el autobús el único medio popular de transportes en el recinto de la ciudad, su reglamentación adecuada y su vigilancia son aspiraciones legítimas de cualquier gobierno municipal. Los acontecimientos de los últimos días, provocados por la extorsión de los monopolizadores del transporte urbano, dieron ocasión a una caudalosa reacción cívica, que le impone al concejo, como única solución legítima la municipalización del servicio (Concejo de Cali, 1946).

Sin embargo, este proyecto sería uno más de varios intentos por mejorar el transporte de la ciudad, que no pasaron del papel y de las intenciones, pues el transporte continuó en manos de empresarios privados y los proyectos por reformarlo tuvieron muy poco éxito.

\section{Planeación y ciudad real}

A partir de la década del cuarenta, el desarrollo del transporte público en Cali estuvo ligado al continuo y acelerado crecimiento perimetral de la ciudad, debido a sus altas tasas de crecimiento poblacional. La proliferación de nuevos barrios y la expansión continua y acelerada de la ciudad requería el suministro de servicios básicos como 
acueducto, energía, vías y transporte; servicios que empezaron a ser suplidos de manera precaria por los mismos habitantes en los barrios informales, y en muchos de los formales que habían sido entregados por programas de vivienda estatal pero sin servicios básicos (Mosquera, 1984).

$\mathrm{El}$ agua se adquiría a través de pilas públicas y la energía por medio de conexiones realizadas por los mismos habitantes; asimismo, la precariedad de las vías dificultaba la comunicación de los habitantes de los barrios informales y periféricos con la ciudad formal. Sin embargo, dichos sectores, a pesar del problema que representaban para la ciudad, eran también su principal fuente de mano de obra, por lo cual era necesario garantizar su movilidad hacia el centro de la ciudad y las zonas industriales. Esta necesidad se hace evidente en el Decreto No 492 de 1964: «Por medio del cual se establece un estatuto municipal sobre el sistema de asignación prolongación o recortes de las rutas urbanas de buses que funcionan en la ciudad de Cali».

A través de este decreto la Alcaldía aprobó recorridos que iniciaban, terminaban y atravesaban barrios aún no legalizados y los conectaban con la ciudad formal. La mayoría de empresas de transporte ubicaron los sitios de salida de los buses -denominados controles de despacho- en barrios surgidos de manera informal o de reciente creación, muchos de los cuales permanecían aún sin legalizar; lo que evidencia que, así como los habitantes de los nuevos barrios en las periferias dependían para su conexión con la ciudad de las empresas de transporte, la creación y consolidación de las empresas dependió también de la conformación de estos barrios y de su amplia población, lo que les permitía captar un gran número de usuarios, teniendo en cuenta que la mayor demanda del servicio de buses se encontraba precisamente en los nuevos barrios populares conformados por obreros y desempleados que buscaban conectarse diariamente con la ciudad formal.

El documento permite establecer que para 1964 existían en Cali 16 empresas de transporte, 13 de las cuales aparecen con una sola ruta o recorrido aprobado, y las restantes con dos. La empresa Blanco y Negro, fundada en 1964, ubicó sus instalaciones en el norte de la ciudad, en el sector de Menga, lo que le permitía captar usuarios que viajaban a la zona industrial ubicada en el municipio contiguo de Yumbo y residentes en Cali. La empresa Santiago de Cali-Alameda, constituida el 14 de febrero de 1946 según escritura pública No 506 de la Cámara de Comercio de Cali, contaba en 1964 con dos controles de despacho en el sur de la ciudad, uno en el barrio Caldas, de reciente creación, y otro en Meléndez, que había iniciado su poblamiento varias décadas atrás pero que solo fue legalizado en 1965.

Todas las empresas de transporte, excepto Blanco y Negro, se ubicaron en barrios populares. Las más cercanas al centro de la ciudad eran Gris Roja y Amarillo y Crema, que se encontraban hacia la zona oriental en barrios con pocos años de fundación o legalización como Villa Colombia y La Base. La empresa Roja y Crema se instaló cerca al Bosque 
Municipal, en zona montañosa, donde ya había empezado el poblamiento de sectores informales como Terrón Colorado. La empresa Santa Rita se encontraba en Calima, barrio alejado del área continua de la ciudad. La empresa Gris San Fernando constituida el 31 de enero de 1950, se situó en la ladera occidental, entre los barrios Belisario Caicedo que inició su ocupación en 1952 y el barrio Siloé que empezó a ser poblado desde las primeras décadas del siglo XX; los dos barrios fueron legalizados en 1964 (Ruiz, 2014). Las empresas Verde Bretaña, creada en 1949, y Verde San Fernando, en 1950, ubicadas en el sector suroriental, cubrieron barrios como El Guabal, Cristóbal Colón y Primero de Mayo, surgidos de tomas populares de terrenos y legalizados en la década del 60.

Por último, los barrios creados luego del desecamiento de la zona oriental de Aguablanca a finales de los años 50, concentraron el mayor número de empresas de transporte. En el barrio Alfonso López, que inició su poblamiento en 1961 a través de labores de un centro pro-vivienda, se encontraban las empresas Puerto Mallarino, Verde Plateada-Villa Colombia y Florida-Cali (Papagayo). Contiguo al sector de Unión de Vivienda Popular, surgido bajo la misma modalidad del anterior, encontramos los controles de Roja Villanueva Belén, Río Cauca, Puerto Mallarino y Azul Crema, al igual que Azul Plateada cuya ruta iniciaba dentro del mismo barrio.

Para 1980 varias empresas habían desplazado sus instalaciones mucho más al oriente, en comparación con la ubicación que tenían en 1964; tal es el caso de Verde San Fernando, Gris Roja, Azul Crema y Verde Plateada. La empresa Gris San Fernando subió un poco más hacía la montaña de Siloé. La empresa Alfonso López, de reciente aparición, se ubicó en el barrio de su mismo nombre. Crema y Verde, que surgió de la división en la empresa Verde Bretaña, aparece instalada en la ladera occidental, sector de Meléndez (ver mapa 2).

Lo anterior evidencia que, cuando muchos barrios aún no habían sido legalizados o apenas iniciaban este proceso, ya contaban con rutas de buses legalmente constituidas y aprobadas por la Alcaldía. Por ello, en varios sectores de la ciudad los buses constituyeron el primer servicio legal que sus habitantes pudieron adquirir, antes del acueducto, la energía, las vías y la inclusión de sus barrios como parte de la ciudad formal.

\section{Las dos ciudades}

A partir de la década del 70, con la reforma constitucional de 1968, la planeación en el país sufrió varios cambios que permitieron pasar de la formulación de planes que se limitaban a regular el crecimiento físico de la ciudad, a planes de desarrollo que involucraban el análisis y planificación de sus aspectos socio-económicos, así como las estrategias que permitieran mejorar la calidad de vida de sus habitantes. Estos cambios llevaron a la creación de la Oficina de Planeación Municipal de Cali en 1969, y a la formulación del Plan General de Desarrollo de Cali y su Área Metropolitana 
MAPA 2 Cambios en ubicación de terminales de buses entre 1964 y 1980

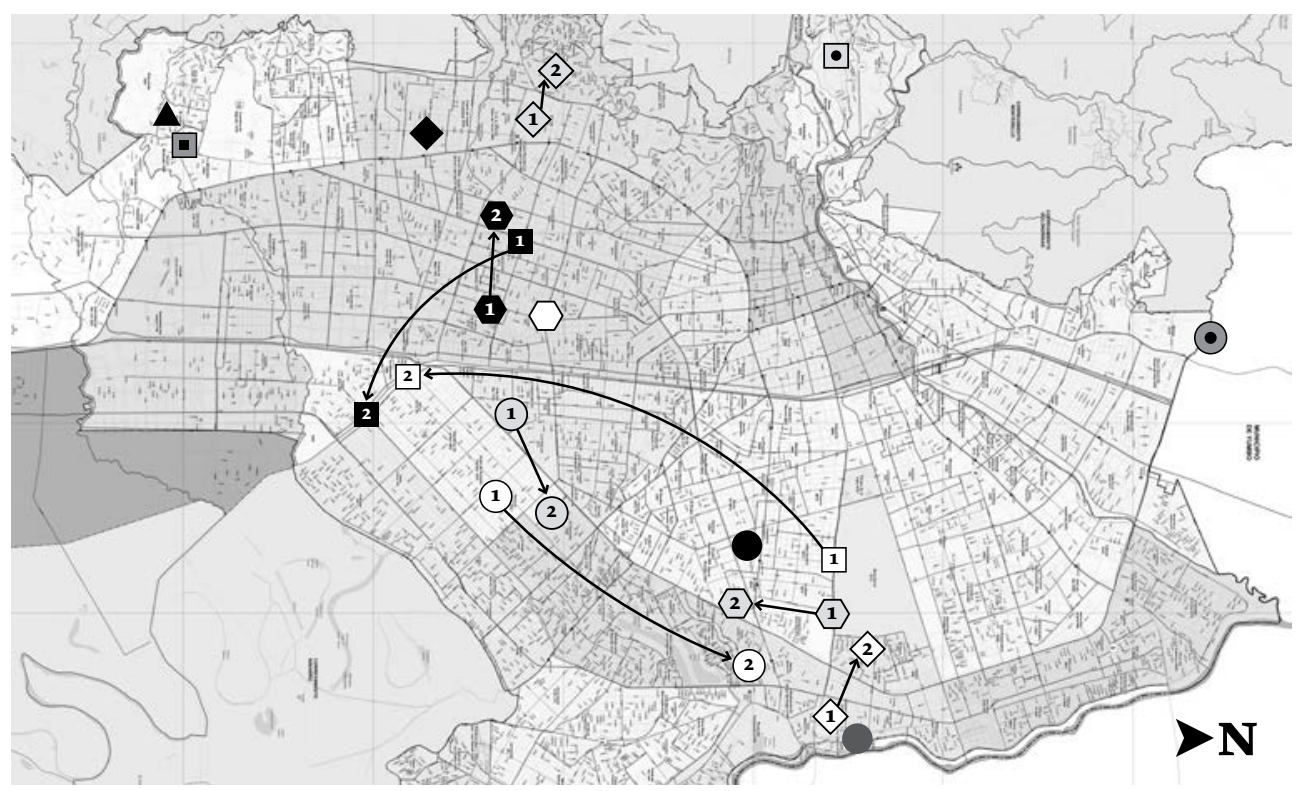

\begin{tabular}{|c|c|c|c|}
\hline Ubicación & Línea o empresa & $\begin{array}{l}\text { Dirección del control } \\
\text { en } 1964(1)\end{array}$ & $\begin{array}{l}\text { Dirección del control } \\
\text { en } 1980(2)\end{array}$ \\
\hline & Amarillo Crema & Suroeste de la ciudad / Zona «Zumba» & Calle 5o, B/ Nueva Floresta \\
\hline & Alfonso López & No existía la empresa & Carrera 7a Cll.82, B/ Alfonso López \\
\hline & Azul Crema & Calle 27 Cr.44, B/ La Independencia & Carrera 41a Cll.3o, B/Antonio Nariño \\
\hline & Azul Plateada & Calle 40 con Carrera 34 & Calle 73 Cr.2o, zona de Aguablanca \\
\hline$\Omega$ & Blanco y Negro & Menga & Sin cambio \\
\hline & Florida Cali-Papagayo & Carrera 8 con Calle 63 , Puerto Mallarino & Carrera 7c Cll.69 \\
\hline & Coomepal & No existía la empresa & Calle 5 a con Carrera 73 \\
\hline & Gris San Fernando & Calle 30 Sur, B/ Belisario Caicedo & $\begin{array}{l}\text { Diagonal } 51 \text { Cll.7a Oeste, B/ Lleras } \\
\text { Camargo }\end{array}$ \\
\hline & Verde San Fernando & Calle 12 Cr.41, B/ El Guabal & Calle 40 Cr.5o, B/ Mariano Ramos \\
\hline$\bullet$ & Crema y Rojo & Bosque Municipal & Sin cambio \\
\hline$\square$ & Crema y Verde & Hacía parte de Verde Bretaña S.A. & Carrera 94 Cll.4a, B/ Meléndez \\
\hline 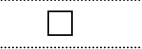 & Gris Roja & Carrera $7 \mathrm{~B}$ con Calle 51 & Carrera 50 Cll.42, B/ Mariano Ramos \\
\hline A & $\begin{array}{l}\text { Santiago de Cali- } \\
\text { Alameda }\end{array}$ & Barrio Meléndez & Sin cambio \\
\hline & Verde Bretaña & Calle 17 Cr. 41 & Calle 10 Cr.52, Autopista Sur \\
\hline & Verde Plateada & Calle 60 manzana 34 , B/ Alfonso López & Calle 57 Cr. 27 \\
\hline$\square$ & Villanueva Belén & Carrera 45 Cll.27 & Sin cambio \\
\hline
\end{tabular}

Fuente: Elaboración propia a partir de datos tomados de: Alcaldía de Cali, Decreto 492, Octubre 10 de 1964) y (Oficina de Planeación Municipal de Cali, Pideca, 1980).Plano tomado de la Subdirección de POT y Servicios Públicos, Departamento Administrativo de Planeación, Alcaldía de Cali.. 
1970-1985-2000-PGD-en 1971, que tenía entre sus principales pretensiones, según el alcalde Carlos Holguín, la disminución de la brecha socio-económica existente entre las zonas periféricas y el resto de la ciudad.

Asimismo, en 1970 se estableció en la ciudad la sede regional del Instituto Nacional de Transporte (INTRA) y en 1972 fue creada la Secretaría de Transporte Municipal, con la cual la Administración Municipal pretendió controlar un sector que al igual que la ciudad crecía aceleradamente y con poco control. A estas dos entidades se asignaron funciones relacionadas con el control del transporte público, a lo que se sumó el establecimiento del subsidio al transporte público por parte del Gobierno Nacional en 1971, para evitar cobros exagerados a los usuarios del transporte.

Según el PGD, una de las principales problemáticas de la ciudad, debido a su forma de crecimiento, eran los «flujos encontrados de desarrollo urbano»: uno de características centrípetas o centralismo físico-funcional y otro centrífugo o de expansión urbana. El flujo centrípeto era causado por la ubicación de la mayoría de servicios y lugares de trabajo en el centro de la ciudad; a pesar del crecimiento, la ciudad seguía funcionando con el modelo antiguo, en el cual todas las vías municipales e incluso las regionales convergían al centro. El flujo centrífugo era generado por el crecimiento incontrolado de la ciudad desde la década del 40, que había llevado a la obsolescencia de sus servicios comunales y de su infraestructura.

Estas dos situaciones significaban el aumento de los problemas de tráfico y las dificultades de movilidad que sufrían los ciudadanos Estos dos fenómenos eran, según el Plan, consecuencia de la falta de estrategias globales y de la toma de decisiones aisladas; por ello se recomendaba descentralizar el comercio y los servicios comunales a las zonas periféricas (PGD, 1971).

Sobre el transporte en modalidad de buses, el PGD indicaba una alta dependencia de la ciudad hacia este servicio, debido al bajo nivel de ingresos de la mayoría de ciudadanos: el 83\% de las familias tenían ingresos menores a \$3.500. Los datos recopilados evidenciaron, además, una estrecha relación no pavimento-bajos ingresos. Según la división de la ciudad en siete circuitos, planteada en el PGD, los sectores que representaban los menores ingresos económicos por familia eran precisamente los que carecían casi por completo de vías pavimentadas.

Con estos datos, el Plan concluía que el mal estado de las vías encarecía la operación de los buses, porque incrementaba los tiempos de viaje y deterioraba los vehículos. Señalaba también que las rutas carecían de lógica, con recorridos muy extensos, desgastando innecesariamente los vehículos; también sobrecargaban algunas vías como la Carrera 15 (centro de la ciudad), por la cual transitaban 25 de las 27 rutas existentes en 1969. Estas afirmaciones del PGD desconocían el sentido que las rutas tenían para los transportadores; lo que para los expertos en transporte representaba un sin sentido, para los transportadores (conductores y dueños de empresas) representaba la opor- 
tunidad de transportar el mayor número de pasajeros, obteniendo la mayor ganancia posible en cada recorrido. La falta de lógica era, en realidad, la lógica económica de las empresas de transporte.

Algunas de las recomendaciones del PGD para mejorar el funcionamiento de los buses fueron: Tratar de establecer rutas con recorridos directos, impedir que los buses entraran al centro de la ciudad, establecer paraderos cada 200 metros e impedir que los buses se detuvieran en cualquier lugar. A pesar de estas recomendaciones, el Plan no precisaba las estrategias que se debían seguir para la organización del transporte, por lo cual, como señala Bonilla (2012), el PGD terminó siendo más analítico que propositivo:

Este enfoque, si bien necesario para corregir el sesgo eminentemente físico de los planes reguladores y pilotos anteriores, adquiere un énfasis fuerte hacia los diagnósticos y su respuesta en torno de las soluciones urbanísticas específicas se diluye en lo cuantitativo sin mayor precisión espacial. A partir de aquí se hará más evidente el divorcio entre el andar urbanístico y la generalidad de los planes (2012).

El Plan también asumió que para el año 1985, el nivel de viajes diarios por persona dentro de la ciudad se mantendría en un promedio de 1,6. Aunque la población aumentaría y las distancias también, los viajes al centro de la ciudad se reducirían, debido a la ubicación de áreas comerciales e industriales en zonas próximas a las áreas residenciales. Además, afirmaba que no habría un gran incremento de vehículos particulares per-cápita; por el contrario aumentarían las vías pavimentadas y las mejoras en la red vial, lo que optimizaría el sistema de transporte público haciendo más eficientes y convenientes sus rutas. También proyectó la construcción de un sistema de Tren Metropolitano que conectaría los municipios de Jamundí, Yumbo y Palmira con Cali, pero advirtió que este aún no era necesario: «La ciudad de Cali no precisará de una solución masiva en el transporte colectivo hasta después del periodo 1980-1985 cuando la población sobrepase los 2.00o.ooo de habitantes» (PGD, 1971).

Del PGD, el Concejo el Plan Vial solo aprobó varias obras de infraestructura, impulsadas además por la realización en la ciudad de los VI Juegos Panamericanos, entre las que se encuentran: Construcción de la Avenida Nueva Granada, parte de la Avenida Tequendama, Avenida Guadalupe, Avenida Los Cerros, Avenida Saavedra Galindo, parte de la Avenida Simón Bolívar y la funalización de la Autopista Sur-oriental (Figueroa, 2013). Aunque las obras realizadas provocaron transformaciones urbanas importantes, solo se limitaron al aspecto físico de la ciudad, por lo cual la pretensión de convertir el PGD en instrumento de políticas públicas en los aspectos económicos, sociales y administrativos, no tuvo éxito (Bonilla, 2012).

La promesa del alcalde Carlos Holguín, de disminuir la brecha socio-económica entre los habitantes de la ciudad no se cumplió; por el contrario, la división establecida con 
la nueva Autopista Sur-oriental, terminó por consolidar un modelo de distribución del espacio urbano denominado las dos ciudades: «la de los integrados y la de los excluidos en términos socio-espaciales» (Vásquez, 2001). En esta división socio-espacial tomó mayor relevancia el transporte público, como mecanismo mediante el cual las dos ciudades se conectaban e interrelacionaban.

\section{Diagnósticos sobre el transporte público}

En 1973 el Intra publicó el Estudio de Reestructuración de Rutas Urbanas para Cali. En los cinco informes publicados se analizaron aspectos como la estructura física, socio-económica y poblacional de la ciudad; los aspectos administrativos y financieros de las empresas de transporte; los indicadores de origen y destino de los viajes dentro de la ciudad; y se hizo un inventario del sistema de transporte público de la ciudad. El quinto informe estaba dedicado a ofrecer alternativas y recomendaciones al transporte de la ciudad, sin embargo es el único del que no se hallan ejemplares en la actualidad.

A pesar de esta ausencia, los otros cuatro tomos ofrecen amplia información sobre la situación del transporte en esta época de manera más detallada que el PGD; principalmente el informe $\mathrm{N}^{\circ}$ 2, que hizo un inventario del sistema de transporte en la modalidad de buses. El objetivo del documento fue establecer los recorridos de los buses en cada una de las 32 rutas existentes en 1972, medir el tiempo de los recorridos, las condiciones de las vías, establecer el estado de los vehículos y las formas de operación de las empresas. Todo esto con el fin de hacer las recomendaciones necesarias para mejorar el sistema de transporte público en la ciudad, que era una de las funciones del Intra.

Luego de recopilar esta información, el análisis arrojó las siguientes conclusiones: Se evidenciaron diferencias entre las rutas autorizadas y las recorridas, sobre todo en los barrios populares. Esta práctica obedecía, según el informe, no a la búsqueda de calles pavimentadas sino a la búsqueda de pasajeros cerca a sus domicilios, lo que hacía que los buses realizaran muchas vueltas por un mismo sitio; práctica que se debía eliminar para agilizar el transporte. Así mismo, la tendencia de los buses era pasar lo más cerca posible al centro, para dejar al usuario muy cerca de su destino. Situaciones que se debían modificar y para lo cual señalaba el Intra, era necesario «educar al usuario y hacerlo comprender la conveniencia de un transporte rápido aunque haya necesidad de caminar más cuadras [...] ya que esto va a resultar en beneficio de él al disminuir el tiempo de viaje total» (Instituto Nacional de Transporte, 1973). También señala el informe que muchos de los recorridos que se realizaban no estaban autorizados, pero que obedecían en unos casos a la necesidad de encontrar pasajeros y en otros a la necesidad de agilizar el tiempo del recorrido. 
Estas tendencias en los recorridos permiten evidenciar una alta operación de los buses por fuera de las rutas aprobadas, con lo cual se puede afirmar que, aunque las empresas y rutas estaban legalmente constituidas, en la práctica actuaban, en un alto porcentaje, por fuera de lo permitido por las autoridades de tránsito para incrementar sus ganancias. Estas formas de operar favorecían la práctica de los usuarios de buscar o esperar las rutas que los recogieran y dejaran más cerca de sus lugares de origen y destino, con lo cual las empresas de transporte, bajo una lógica de mercado, desestimulaban la aceptación de los ciudadanos a medidas como la peatonalización del centro o la instalación de paraderos exclusivos que evitaran a los buses detenerse constantemente en busca de pasajeros.

Otra de las observaciones del informe fue la necesidad de adecuar las vías para evitar los recorridos «tortuosos» y agilizar los tiempos, principalmente en el sector oriental y suroriental de la ciudad: gran parte de los recorridos se hacían por vías sin pavimentar. Sin embargo, señalaba el informe que todas las zonas de la ciudad estaban adecuadamente atendidas por el servicio de buses; lo que muestra que el pavimento no era un factor de decisión a la hora de establecer el recorrido de los buses. Por el contrario, si tenemos en cuenta la relación no pavimento-bajos ingresos establecida en el PGD y la dependencia de los sectores populares hacia este servicio, la precariedad de algunas zonas podía significar el éxito de algunas rutas al tener mayor oportunidad de captar usuarios.

En los otros tres informes del Estudio de reestructuración de rutas, el Intra recomendaba limitar el área de expansión de la ciudad y densificar las áreas centrales, controlando principalmente el uso residencial en la zona oriental. La estructura administrativa de las empresas de transporte se calificaba como inadecuada y poco funcional. Según el Intra, a pesar de la desorganización financiera de las empresas, estas subsistían debido a la rentabilidad del negocio del transporte en la ciudad. Para 1972, los ciudadanos realizaban diariamente 1.200.ooo viajes dentro de la ciudad con diferentes propósitos, de los cuales 900.000 eran realizados en bus, el $75 \%$ del total de viajes diarios. La mayoría de estos viajes eran realizados desde la zona suroriental y oriental hacia el centro. A pesar de las recomendaciones y el diagnóstico realizado por el Intra, la propuesta de reestructuración, que era el máximo objetivo del estudio, se desconoce por la desaparición del Informe No 5 .

Luego del estudio realizado por el Intra, encontramos un diagnóstico realizado por la Secretaría de Tránsito en 1978: Evaluación transporte público urbano en Cali. Lo primero que se evidencia es que entre la realización del estudio del Intra y este nuevo informe, la situación del transporte público no había tenido cambios significativos. Aunque las problemáticas señaladas en el estudio anterior se conservaban, el nuevo estudio era mucho más crítico respecto a la forma en que se venía manejando la operación de las empresas y el papel del Intra, ya que en muchos casos había duplicidad de funciones entre la Secretaría y el Intra: «La duplicidad de funciones ha originado que las empresas 
evadan con facilidad infracciones de operación, escudándose, cuando le conviene, en cualquiera de las entidades Intra Valle, Datt Valle o Tránsito Municipal» (Secretaría de Tránsito y Transporte Municipal de Cali, 1978).

Las principales conclusiones de la Secretaría fueron que el sistema de transporte urbano se caracterizaba por «un ánimo exclusivo de lucro», sin tener en cuenta la calidad del servicio. Las rutas habían nacido sin planificación, ya que las empresas, de acuerdo al crecimiento de la ciudad, iban solicitando nuevas rutas o la prolongación de las existentes. Es decir que, aunque en teoría la decisión sobre los recorridos de los buses le correspondía al Intra, en la práctica eran los directivos de las empresas de transporte quienes decidían hacia dónde enviar las rutas. Decisiones que posteriormente eran aprobadas por el Intra, pero que en principio tomaban los empresarios del transporte, quienes según el mismo informe eran en su mayoría personas con conocimientos empíricos a las cuales les interesaba únicamente sus ganancias (Secretaría de Tránsito y Transporte Municipal de Cali, 1978).

A pesar de todos los temas expuestos en el Estudio de reestructuración de rutas del Intra y en la Evaluación del transporte público urbano de la Secretaría de Tránsito, los dos omitieron temas como el análisis de las deficiencias del transporte en las zonas de ladera, centrándose en el análisis del Oriente de la ciudad; y el análisis de las formas de contratación de los conductores, como uno de los factores de deficiencia en el transporte. Los conductores obtenían sus ganancias de acuerdo al número de pasajeros que lograban captar diariamente, lo que originó la competencia de buses por las calles de la ciudad, buscando añadir un pasajero más a la ganancia del día; problemática conocida como «la guerra del centavo» y causante de la mayoría de accidentes de buses en las vías.

El primer documento que analizó las condiciones laborales de los conductores de bus en la ciudad fue el Plan integral de desarrollo de Cali y su área metropolitana-Pideca-, publicado en 1980, y que vino a remplazar al PGD. Condiciones laborales que además, redundaban en un mal servicio a los ciudadanos:

No tienen salario fijo.

Tienen pocas prestaciones sociales.

Su nivel cultural es bajo.

Además de conducir, ejecutan otras labores como cobrador y mecánico.

No tienen jornada fija de trabajo y por lo general esta es inhumana.

No existe la carrera de conductor, cualquier persona consigue un pase y puede manejar un vehículo público.

No tienen bien definido el patrón. [Jefe]

Existe la tendencia de asignar un conductor a un vehículo. 
Tienen un sistema de pago a base de centavos por pasajero transportado, altamente inconveniente.

Son los que tienen una jornada larga más constante.

Se les acusa de ser los únicos causales de los problemas de tráfico (Pideca, 1980)

Señala Samuel Jaramillo (1985) que la mayoría de los conductores no eran propietarios sino asalariados; las funciones de las empresas eran mínimas y cumplían muy poco con su papel de regular el servicio; por el contrario, el beneficio que recibían al controlar las rutas llevó a una relación de dependencia de los propietarios de buses con las empresas, que permitió el cobro de rentas y la creación de mercados cautivos para los insumos de los vehículos. A esto se añadió el gran atraso de las condiciones laborales de los conductores, ya que los vínculos se establecían no con la empresa, sino entre el trabajador y el propietario del vehículo:

Sus jornadas son enormemente prolongadas, tienen muy poca estabilidad, el trabajo es muy intenso y desgastador, pues deben simultáneamente cobrar y conducir en un tráfico endemoniado, compitiendo con sus colegas por los pasajeros de los cuales depende su salario. Carecen en la práctica de prestaciones sociales como la jubilación, horas extras, no reciben ingresos cuando el vehículo se avería, y por el contrario deben realizar gratuitamente tareas de reparación y de mantenimiento. Y sin embargo [...] el salario global que estos conductores pueden reunir es comparativamente alto, entre dos y dos y media veces el salario mínimo (Jaramillo, 1985).

Por su parte, las leyes vigentes sobre contratación laboral en el país señalaban:

El contrato de trabajo verbal o escrito, de los choferes asalariados del servicio público, se entenderá celebrados con las empresas respectivas, pero para efecto de pago de salarios, prestaciones e indemnizaciones, las empresas y los propietarios de los vehículos, sean socios o afiliados, serán solidariamente responsables. (Congreso de Colombia, 1959).

En la práctica, ni la empresa ni el dueño del vehículo asumían las responsabilidades de ley. Las ganancias, mayores al promedio, hacían que los conductores prescindieran de sus derechos laborales y que se normalizaran las prácticas de contratación que favorecían las ganancias de las empresas en detrimento de la calidad de vida de los conductores. Todo ante la indiferencia de las autoridades nacionales, quienes optaron por no enfrentarse a los empresarios del transporte que adquirían cada vez mayor poder en el país. Poder que les permitía incluso, manejar abiertamente un esquema de contratación contrario al ordenado por las leyes vigentes. 
Las condiciones en que operaban las empresas eran favorecidas por el número creciente de usuarios, debido a la tasa de crecimiento de la ciudad. Según el Pideca, las continuas obras de infraestructura y servicios causaban un efecto multiplicador de la población que en su mayoría llegaba a ocupar los sectores populares e informales de la ciudad. Estas zonas continuaban significando para las empresas, las mayores oportunidades de obtener rutas. La ubicación que habían adquirido la mayoría de terminales, en la zona oriental, significó para sus habitantes una garantía de comunicación y de inserción en las oportunidades económicas y laborales que ofrecía la ciudad formal. Según señala Aprile-Gniset:

La aculturación urbana de un individuo es proporcional a las oportunidades de inserción que le ofrecen tanto la sociedad de la ciudad como sus estructuras económicas. [...] Limitándonos a la vivienda, por ejemplo, vemos como muchos de los nuevos citadinos en razón de un «accidente» que cambió la trayectoria de su vida, están residenciados en un lugar extraño, insólito, con el cual no se sienten ni identificados ni solidarizados: residen muy a menudo en unos lejanos suburbios indecisos, en un espacio en tránsito desde lo rural hacia lo urbano, pero que no han logrado aún su integración a la ciudad. Para la población de estas regiones urbanas fronterizas, la ciudad sigue siendo simbolizada por altas torres blancas en el centro, que se ven allá, al fondo, surgiendo de la horizontalidad, a donde se llega después de un largo recorrido, en un bus destartalado (1992).

Esta conexión, garantía de oportunidades laborales y económicas, era uno más de los servicios que los barrios requerían. Como venía ocurriendo desde décadas atrás, el transporte era uno de los primeros servicios en llegar a los barrios informales y de reciente ocupación, a pesar de la falta de organización y el ánimo de lucro de los transportadores, o debido a ello. El caso del barrio El Retiro es muestra de ello:

\footnotetext{
Al inicio se sufrió inhumanamente, las gentes más afectadas fueron las del barrio El Retiro, porque los terrenos entregados eran lagunas, cuando llovía era el primero en inundarse, donde les tocó prácticamente hacer el relleno de todo el barrio. Se alumbraban con vela, en el primer año no había alcantarillado, eran solamente letrinas y sanitarios que se colocaron en el canal de la calle 48 [...] En materia de transporte, no eran sino dos las empresas que prestaban el servicio (Azul Crema y Alfonso López), en muchas ocasiones el transporte quedaba suspendido por el mal estado de las vías cuando llovía (Centro de Administración Local Integrada Comuna 15, 2003).
}

Las historias de cómo se conformaron los barrios surgidos en condiciones precarias, evidencian la importancia de los buses en los procesos de configuración de estas zonas periféricas, contribuyendo incluso, en cierta forma, a legitimarlas como parte de la ciudad. 


\section{Soluciones a los problemas del transporte público}

A partir de la década del 70, el transporte público colectivo en el país estuvo marcado por la creación, por parte del gobierno nacional, del subsidio al transporte urbano, a través del Decreto $\mathrm{N}^{\circ} 1277$ de 1971 (Presidencia de la República, 1971), medida que definió la actuación de las empresas de transporte y su relación con el Estado. El subsidio se pagaba por cada vehículo en funcionamiento y tenía como finalidad, en un principio, cubrir el sobrecosto de la gasolina para que esta cifra no fuera trasladada a los usuarios en aumentos del valor del pasaje.

El subsidio se conservó durante dos décadas, ya que los costos de operación de las empresas arrojaban que, con el valor en el que se mantenía el pasaje, el negocio generaba pérdidas. Por lo cual, para no trasladar todo el aumento al usuario el gobierno compensaba con el subsidio una parte del valor del pasaje. El subsidio se pagaba directamente a las empresas por cada vehículo afiliado de acuerdo a sus días de servicio en el mes: de 1 a 10 días no había derecho a subsidio; de 11 a 20 días se pagaba el 50\% del subsidio y de 21 a 30 días se pagaba el 100\% del subsidio (Presidencia de la República, 1972). Por día de servicio se entendía la realización de mínimo tres recorridos completos de una ruta asignada. Para el cobro, el gobierno suministraba un formulario que debía ser llenado por la empresa de transporte de acuerdo a las planillas de control y despacho de los vehículos. Modalidad que generó polémica ya que se hablaba de planillas alteradas, al marcar recorridos que los buses no realizaban. Otra modalidad era la circulación del bus sin importar la poca afluencia de pasajeros; aunque no se percibieran ganancias en el recorrido, el dueño del vehículo y la empresa salvaban el cobro del subsidio.

A inicios de los años 8o, el Gobierno Nacional, a través del Intra, inició el desmonte progresivo de los subsidios al transporte, justificado en las irregularidades que se presentaban en su cobro y en la mala calidad del servicio. Las críticas a los subsidios habían aumentado, debido a las continuas alzas que los transportadores exigían al Gobierno Nacional y a las denuncias sobre los posibles fraudes que se estaban cometiendo en su cobro. A esto se sumaba que muchos transportadores optaron porque sus buses hicieran únicamente los recorridos requeridos para obtener el subsidio, como lo describe el diario El Tiempo:

En todas las ciudades del país se observa diariamente el fenómeno angustioso, especialmente en las horas pico, de largas colas de usuarios esperando movilizarse [...] Como es del conocimiento general existe gran cantidad de vehículos que no son trabajados normalmente debido al criterio erróneo de los propietarios de trabajar únicamente el tiempo requerido para el pago del subsidio lo cual conlleva al entorpecimiento del servicio del transporte de pasajeros en la parte urbana en diferentes ciudades del país (El Tiempo, 1982). 
Las posiciones estaban claras, el gobierno solicitaba que las empresas de transporte mejoraran el servicio, adquirieran mejores vehículos, disminuyeran el sobrecupo y colocaran en funcionamiento todo el parque automotor del que disponían. Los transportadores argumentaban que el subsidio solo compensaba tres recorridos y el resto se trabajaba a pérdida, por lo cual, para obtener ganancias, los buses debían ir llenos y adquirir vehículos nuevos no era un buen negocio, ya que con los viejos se obtenía la misma ganancia. Por ello, en 1982 el Intra creó una nueva modalidad de transporte en el país: el transporte sin subsidio (TSS). Con ello, las empresas cobrarían la tarifa plena que les garantizaba ganancia en todos los recorridos y el Gobierno disminuiría el gasto en subsidios.

Mediante Resolución No 342 de 1982, el Intra dispuso las características que debían cumplir los buses que prestarían el nuevo servicio: solo se aceptaban vehículos modelo 1981 y posteriores, debían tener silletería de lujo, puertas más amplias, debían contar con un aviso frontal luminoso con las letras TSS y ser pintados de color amarillo y verde para diferenciarse claramente de los buses subsidiados. Los TSS solo podían llevar 20 personas de pie, para evitar el sobrecupo. Las empresas podían cambiar su parque automotor y remplazarlo completamente por buses que cumplieran con las nuevas características o podían seguir funcionando en las dos modalidades: TSS y subsidiado (Instituto Nacional de Transporte, 1982). Sin embargo, para este momento ya se dudaba de los efectos positivos de la medida, teniendo en cuenta que el desmonte de los subsidios significa, principalmente, el aumento en las tarifas a los usuarios:

Sin embargo, a pesar de las distorsiones y de los malos usos a que da lugar por parte de algunos transportistas, el subsidio es la única medida viable para que los colombianos puedan ir de un lado a otro sin que les cueste más de lo que pueden pagar. [...] El TSS es el ejemplo de lo que pasaría si prosperara el intento de desmontar este sistema: son \$7.5O más que salen del bolsillo del pasajero, mientras que lo único que cambia del bus es el color (Revista Semana, 1982).

Aun así, continuó el desmonte del sistema de subsidios. A su llegada a la presidencia en 1983, Belisario Betancur, mediante el Decreto No 3615, estableció una rebaja en los subsidios entregados a cada vehículo y lo condicionó al modelo de fabricación de los buses: a mayor antigüedad menor subsidio. Los buses más nuevos, modelos entre 1974 y 1978, que funcionaran con sistema diésel, recibirían mayor subsidio que los de gasolina. Los recorridos diarios que debía realizar cada bus para obtener el subsidio también se aumentaron dependiendo del tamaño de las ciudades; para Cali fueron aumentados de tres a cinco.

También se anunció que a partir el 1 de enero de 1984, mediante reglamentación del Intra, los buses de modelos posteriores a 1978 serían retirados del programa de 
subsidios e ingresarían al de TSS. Menos de un año después, el presidente Betancur emitió el Decreto No 2775 que retiraba del sistema de subsidios a los buses de modelos entre 1974 y 1977 y los incluía en el sistema TSS. Las condiciones iniciales para el cobro de tarifa plena por parte de los TSS habían desaparecido y las mejoras en el servicio nunca llegaron. Los buses con subsidio eran escasos y las tarifas subían. Los buses verde-amarillos continuaban aumentando.

De la misma época es el proyecto de construcción de un sistema de transporte masivo, para lo cual el Concejo de la ciudad, mediante Acuerdo № 41 de 1979, impulsó la creación de la Promotora de Transporte Masivo del Valle (Protrans). Un año después, para impulsar los proyectos sobre transporte, el Concejo destinó la vía del ferrocarril que atraviesa la ciudad de sur a norte como área de reserva para el futuro sistema de transporte masivo.

Cabe aclarar que la idea de construir un sistema masivo de transporte ya se estaba discutiendo en Medellín y Bogotá. Desde la década del 70, en Medellín se había reservado un área de 60 metros a lado y lado del río para su construcción; y en 1977, un proyecto de Tren Metropolitano fue incluido en el Plan Metropolitano. Ese mismo año, la ciudad de Bogotá inició estudios para la construcción de un Metro con asesoría de técnicos franceses y un año después constituyó la empresa Metro (Acevedo, Castañeda y Salazar, 1993). En este ambiente surge la idea de construir un sistema masivo de transporte para Cali, aunque finalmente la disputa por los recursos nacionales para el proyecto se dio entre las otras dos ciudades. La llegada de un antioqueño a la presidencia del país terminó por inclinar la balanza hacía Medellín (Acevedo, Castañeda y Salazar, 1993).

En 1983, Protrans publicó el Estudio de Posibles Soluciones para el Transporte Público Masivo en el Área del Valle Geográfico del Rio Cauca. Estudio realizado por Protrans y la firma italiana Ansaldo Trasporti S.P.A., en el marco del Acuerdo de Cooperación técnica internacional entre Colombia e Italia (1971) que permitió el aporte financiero del Ministerio de Relaciones Exteriores del país europeo al proyecto. Con la ayuda técnica y económica de la firma italiana, Protrans desarrolló cuatro propuestas de intervención de la red ferroviaria en el departamento. Todas las propuestas fueron analizadas a partir de sus costos operativos e ingresos de acuerdo a la demanda de transporte que se proyectaba hasta el año 2000. El análisis concluyó que ninguna de las alternativas arrojaba resultados económicos aceptables. Por ello, la decisión se orientaba hacia una inversión de «costo mínimo» que justificara económicamente el funcionamiento del ferrocarril, de bajo costo energético, estratégica para el transporte pesado del país y susceptible de ser utilizada para el transporte de pasajeros. Finalmente, no fue llevado a cabo ningún proyecto y Protrans desapareció.

En 1986, con la aprobación de la elección popular de alcaldes y la asignación de mayores funciones a los municipios, fue eliminado el Intra y sus funciones pasaron a los gobiernos municipales. Asimismo, para 1989, la Corte Constitucional declaró inexequi- 
bles el Decreto 1277 de 1971 y el Decreto 588 de 1978, que regulaban los subsidios a los transportadores. Los subsidios se intentaron revivir con la Ley $2^{a}$ de 1989 , pero esta fue demandada señalando que el Congreso estaba decretando «gratificaciones» del poder público a particulares y que el subsidio no estaba sujeto a planes ni era un derecho de los transportadores. La demanda fue aceptada y la Ley declarada inexequible, con lo cual el subsidio llegó a su fin (Corte Suprema de Justicia de Colombia, 1989).

Durante la década del 80, con la entrada paulatina de buses a la modalidad TSS, las empresas caleñas fueron unificando el color de los buses que se iban incluyendo en esta modalidad, que debían estar pintados de verde y amarillo. Aunque, según lo ordenado por el Intra, los TSS debían tener otras especificaciones físicas y un modo de operación distinta, en la práctica por lo único que terminaron diferenciándose de los buses subsidiados fue por el color.

A inicios de los 9o, cuando desapareció el subsidio, la mayoría de buses de la ciudad habían adquirido los colores del TSS. Por ello, mediante Decreto No o8 de 1993, el alcalde Rodrigo Guerrero ordenó a las empresas adquirir nuevamente para sus vehículos, los colores que habían sido tradicionales hasta la década del 70: «Con el objeto de que los usuarios dispongan de una facilidad visual para distinguir y escoger el servicio deseado sin someterse a las traumáticas confusiones generadas por la uniformidad en los colores distintivos existentes» (Alcaldía de Cali, 1993).

Para adoptar la medida, las empresas tenían un plazo de 18 meses y su incumplimiento podía acarrearles sanciones; los buses que ingresaran por primera vez o los que cambiaran de empresa, debían tener los colores distintivos para que el trámite fuera aceptado por la Secretaría de Tránsito. Sin embargo, los empresarios del transporte presionaron varias veces por la prórroga del plazo para cumplir la medida, por lo cual el tiempo establecido para el cambio terminó ampliándose, a través de sucesivos decretos, hasta 1998. Este es un ejemplo más del poder que llegaron a adquirir los empresarios en las decisiones sobre transporte que se tomaban en la ciudad. Las modificaciones y propuestas en el funcionamiento del transporte público e incluso en el tránsito general de la ciudad debían su éxito o fracaso, en gran parte, a la receptividad que tuvieran en este gremio.

\section{Conclusiones}

El transporte público de Cali fue uno de los sectores que menos cambios sufrió durante el siglo XX, a pesar de los problemas que generó el sostenimiento de un único modelo de transporte público y su operación en manos de sectores privados. Las formas de creación y prolongación de rutas, las condiciones laborales de los conductores y el manejo administrativo de las empresas se conservaron casi sin variación durante la segunda mitad del siglo XX. 
Al igual que los barrios informales, muchas empresas de transporte surgieron y funcionaron sin constituirse legalmente; modalidad que encontraba sustento en las necesidades de la ciudad que crecía física y demográficamente sin encontrar soluciones en los pocos intentos de organización desde la Administración Municipal. La extensión constante e incontrolada de la ciudad en todas sus direcciones, demandaba una solución profunda a las necesidades de movilidad, la cual fue suplida por los empresarios del transporte, con ánimo exclusivo de lucro, y con la connivencia de los concejales y alcaldes de turno que encontraban en las formas de operación de los empresarios privados un escape al problema que se debía enfrentar desde el sector público.

A partir de la década del 70 se proyectaron soluciones para organizar el sector del transporte público; pero el aumento constante de empresas de transporte, el poder que habían adquirido los empresarios, la extensión de la ciudad y la falta de voluntad política para municipalizar el servicio, dificultaron esta tarea. Por su parte, las empresas de transporte lograron responder a las necesidades de movilización de los ciudadanos, y a pesar de las críticas de todos los sectores debido a la mala calidad del servicio, cumplieron un papel importante en la expansión urbana, al brindar a los pobladores de zonas formales e informales el acceso a la ciudad de forma continua y hasta cierto punto eficaz, facilitando las condiciones mínimas para residir cada vez más lejos del centro de la ciudad.

\section{Referencias}

ACEVEDO, J., SALAZAR, C. Y CASTAÑEDA, W. (1993). El metro de Medellin: una ilusión costeada por todos los colombianos. Bogotá: Fonade.

APRILE-GNISET, J. (1992). La Ciudad Colombiana: siglo XIX y siglo XX. Bogotá: Banco Popular.

BONILLA, R. (2012). «Modelos urbanísticos de Cali en el siglo XX: Una visión desde la morfología urbana». En: Loaiza, G. Historia de Cali siglo XX, Tomo I: Espacio urbano, Cali: Facultad de Humanidades/ Universidad del Valle.

FIGUEROA, E. (2013). «Grandes eventos como oportunidades de transformación urbana: los Juegos Panamericanos de 1971 en Santiago de Cali» Tesis de Doctorado, Pontificia Universidad Católica de Chile.

JARAMILLO, S. (1985). «El transporte colectivo en Bogotá: Los límites de la informalidad». En: Transporte y servicios urbanos en América Latina: Actas del Taller de Investigación, Tomo I. Quito: Inrets.

JIMÉNEZ, N. (2014). «Elementos históricos y urbanos en la generación de desastres por inundaciones y deslizamientos en Cali, 1950-2000». Tesis de pregrado, Universidad del Valle, Cali.

MOSQUERA, G. (1984). Morfología, desarrollo y autoconstrucción en Cali: Algunos apartes de la investigación, Cali: Universidad del Valle-Facultad de Arquitectura. 
RUÍZ, A. (2014). «Espacio y poblamiento en la ladera sur occidental de Cali: sector Siloé, décadas de 1910 a 2010». Tesis de pregrado, Universidad del Valle, Cali.

VÁSQUEZ, É. (1980) «Ensayos sobre la historia del desarrollo urbano en Cali». Historia y Espacio (5), $8-63$.

VÁSQUEZ, É. (1995). Retrospectiva urbana y servicios públicos en Cali: 190o-1993. Cali: CIDSE/Universidad del Valle.

VÁSQUEZ, É. (2001). Historia de Cali en el siglo 20: Sociedad, economía, cultura y espacio. Cali: Artes gráficas del Valle.

\section{Documentos institucionales}

ALCALDÍA DE CALI. (1964) Decreto 492 de octubre 10 de 1964 «Por el cual se establece un estatuto municipal sobre el sistema de asignación, prolongación o recortes de las rutas urbanas de buses que funcionan en la ciudad de Cali». Archivo Histórico de Cali/ Fondo Alcaldía, Cali.

CENTRO DE ADMINISTRACIÓN LOCAL INTEGRADA, Comuna 15. (2003). Plan de desarrollo estratégico Comuna 15, Cali: Departamento Administrativo de Planeación- Alcaldía de Cali. [En línea] Recuperado el 10 de octubre de 2015 <http://www.cali.gov.co/publico2/documentos/planeacion/ planterritorial/como8.pdf>

CORTE SUPREMA DE JUSTICIA DE COLOMBIA. (1989). Sentencia 96, Octubre 26 de 1989. [En línea] Recuperado el 12 de Diciembre de $2015<\mathrm{ftp}$ //ftp.camara.gov.co/camara/basedoc/csj_nf/sp/>

COMPAÑIIA PROMOTORA DE TRANSPORTE MASIVO DEL VALLE S.A. ANSALDO TRANSPORTI S.P.A. (1983). Estudio de Posibles Soluciones para el Transporte Público Masivo en el Área del Valle Geográfico del Rio Cauca, Tomo V, Memorias. Cali: Protrans.

CONCEJO MUNICIPAL DE CALI. (1946) Acuerdo 107, julio 29 de 1946 «Por el cual se establece el servicio de buses municipales». Archivo Histórico de Cali/Fondo Concejo, Cali.

CONGRESO DE COLOMBIA. Ley 15, abril 30 de 1959 «Por la cual se da mandato al Estadopara intervenir en la industria del transporte». En: Cifuentes, I. (1986). Legislación del transporte terrestre automotor 1986: Normas vigentes, Bogotá: Presencia.

INSTITUTO NACIONAL DE TRANSPORTE. (1973) Reestructuración rutas urbanas. Informe $\mathrm{N}^{o}$ 2, Cali: Instituto Nacional del transporte-Regional Valle del Cauca.

INSTITUTO NACIONAL DE TRANSPORTE. (1986). Resolución 342, junio 9 de 1982 «Por la cual se crea y reglamenta, en el servicio urbano, el transporte sin subsidio (TSS)». En: CIFUENTES, I. Legislación del transporte terrestre automotor: Normas vigentes, Bogotá: Editorial Presencia.

OFICINA DE PLANEACIÓN MUNICIPAL DE CALI. (1971). Plan general de desarrollo de Caliy suárea metropolitana 1970-1985-20oo, Cali: Oficina de Planeación municipal-Alcaldía de Cali. 
OFICINA DE PLANEACIÓN MUNICIPAL DE CALI. (1980) Plan Integral de desarrollo de Cali y su área de influencia, Documento 17: Situación del transporte. Cali: Alcaldía de Cali.

PRESIDENCIA DE LA REPÚBLICA DE COLOMBIA. (1971). Decreto 1277 del 26 de junio 1971 «Por cual se crea un subsidio de transporte colectivo urbanoy se deroga el Decreto 1255 de 1971». [En línea] Disponible en:<ftp://ftp.camara.gov.co/camara/basedoc\%2015-0711/decreto/1971/decreto_1277_1971. html>

PRESIDENCIA DE LA REPÚBLICA DE COLOMBIA. (1972). Decreto 272, 26 de febrero de 1972 «Por el cual se dictan normas sobre el pago del subsidio al transporte público colectivo urbano». [En línea]. Disponible en:<http://webidu.idu.gov.co:909o/pmb/opac_css/doc_num.php?explnum_id=7442>

SECRETARÍA DE TRÁNSITO Y TRANSPORTE MUNICIPAL. (1978) Evaluación transporte público urbano en Cali. Cali: Secretaría de Tránsito y Transporte Municipal-Alcaldía de Cali.

\section{Publicaciones periódicas}

EL TIEMPO. (1982). «Aprueban plan de buses sin subsidio». Bogotá: Agosto 20 de 1982.p.8.A [En línea] Recuperado el 18 de octubre de 2015. <https://news.google.com/newspapers?id=_KoqAAAAIBA$\mathrm{J} \& \mathrm{sjid}=\mathrm{sF} 4 \mathrm{EAAAAIBAJ} \& \mathrm{hl}=\mathrm{es} \& \mathrm{pg}=1065 \% 2 \mathrm{C} 4005328>$

RELATOR. (1938). «La manifestación anti-fascista ante el cabildo». Cali: Enero 13 de 1938.

REVISTA SEMANA. (1982). «Siempre paga el pasajero», Bogotá: Agosto 30 de 1982. [En línea] Recuperado el 17 de octubre de 2015. <http://www.semana.com/nacion/articulo/siempre-paga-el-pasajero/693-3> 
1948

Diario Relator
1964

Decreto 492 / 1964
1973

Reestructuración rutas

urbanas, INTRA

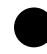

(1) Línea Amarilla

(1) Azul Plateada

(1) Verde Popular Bretaña

(1) Verde San Fernando

(1) Alameda

(1) Gris San Fernando

(1) Roja Estrella

(1) Roja Granada

Empresas: 8

Rutas: 8
(1) Amarillo y Crema S.A.

(1) Azul Plateada

(2) Verde Bretaña S.A.

(1) Cooperativa de Transportes del Valle / Verde San Fernando

(2) Transportes Santiago de Cali S.A. - Alameda

(2) Gris San Fernando Ltda.

(1) Azul y Crema Ltda.

(1) Transportes Verde Plateada Villacolombia

(1) Gris Roja Ltda.

(1) Rojo y Crema Ltda.

(1) Transportes Roja Villanueva Belén

(1) Blanco y Negro Ltda.

(1) Cooperativa de Transportes Puerto Mallarino

(1) Coop. de Transportadores Florida Cali Ltda - Papagayo

(1) Transportes Santa Rita Popular

(1) Transportes Río Cauca S.A.

Empresas: 16 Rutas: 19
(2) Amarillo y Crema S.A.

(2) Azul Plateada S.A.

(4) Verde Bretaña S.A.

(4) Verde San Fernando

(3) Transportes Santiago de Cali S.A. - Alameda

(3) Gris San Fernando Ltda.

(1) Azul y Crema Ltda.

(2) Verde Plateada Villacolombia Ltda.

(1) Gris Roja S.A.

(1) Rojo y Crema Ltda.

(2) Villanueva Belén Ltda.

(2) Blanco y Negro S.A.

(1) Cooperativa de Transportes Puerto Mallarino

(2) Coop. de Transportadores Florida Cali Ltda - Papagayo

(2) Alfonso López S.A.

(1) Cooperativa de Motoristas de Expreso Palmira - Coomoepal

Empresas: 16

Rutas: 33 
* Los años corresponden a la fecha de publicación de la fuente ${ }^{* *}$ Los números entre paréntesis corresponden al número de rutas que operaba la empresa | Nota: Los cuadros en gris corresponden a empresas que sólo aparecen en un periodo.

1980

PIDECA
1993

Manual de rutas

de buses Cali
2000

Diagnóstico del transporte público. Secretaría de Tránsito
(1) Amarillo Crema S.A.

(2) Azul Plateada S.A.

(1) Verde Bretaña S.A.

(3) Crema y Verde S.A.

(5) Verde San Fernando S.A.

(2) Transportes Santiago de Cali S.A. - Alameda

(4) Cooperativa Gris San Fernando

(2) Azul y Crema Ltda.

(4) Verde Plateada S.A.

(1) Gris Roja S.A.

(1) Crema y Rojo Ltda.

(2) Villanueva Belén Ltda.

(8) Blanco y Negro S.A.

(3) Coop. de Transportadores Florida Cali Ltda - Papagayo

(2) Alfonso López S.A.

(2) Cooperativa de Motoristas de Expreso Palmira - Coomoepal

\section{Empresas: 16 Rutas: 44}
(4) Amarillo Crema S.A.
(4) Azul Plateada S.A.
(6) Transportadora Verde Bretaña S.A.
(2) Crema y Verde
(6) Verde San Fernando
(6) Transportes Santiago de Cali S.A. - Alameda
(5) Cooperativa de Transportadores Gris San Fernando Ltda.

(4) Azul y Crema Ltda.

(6) Transportes Verde Plateada Luís Correa y Cia. S. en C.

(4) Gris Roja Ltda.

(4) Crema y Rojo Ltda.

(5) Villanueva Belén Ltda.

(12) Blanco y Negro S.A.

(10) Coop. Integral de Transportadores Florida Cali Ltda - Papagayo

(7) Alfonso López S.A.

(13) Cooperativa de Motoristas y Transportadores Coomoepal Ltda.

(2) Cañaveral

(2) Trans Urbanos Cali S.A.

(5) Pance S.A.

(7) Transportes Recreativos Ltda

(2) Transportes La Ermita

(6) Transportes Montebello

(2) Río Cali S.A.
(6) Amarillo Crema S.A.

(6) Azul Plateada S.A.

(13) Transportadora Verde Bretaña S.A.

(9) Verde San Fernando

(9) Transportes Santiago de Cali S.A. - Alameda

(6) Gris San Fernando S.A.

(5) Azul y Crema Ltda.

(10) Verde Plateada S. en C.

(5) Gris Roja Ltda.

(5) Crema y Rojo Ltda.

(5) Villanueva Belén Ltda.

(17) Blanco y Negro S.A.

(13) Coop. Integral de Transportadores Florida Cali Ltda - Papagayo

(8) Alfonso López S.A.

(20) Coomoepal Ltda.

(3) Transportes Cañaveral S.A.

(2) Trans Urbanos Cali Ltda.

(9) Pance S.A.

(16) Transportes Recreativos

(12) Transportes La Ermita

(11) Transportes Montebello

(4) Río Cali S.A.

(5) Decepaz

Empresas: 23

Rutas: 200 